\title{
Nanohole Array as a Lens
}

Fu Min Huang, Tsung Sheng Kao, Vassili A. Fedotov, and Nikolay I. Zheludev

Optoelectronics Research Centre,

University of Southampton,

SO17 1BJ, UK,

\begin{abstract}
Here we theoretically demonstrate that a quasi-crystal array of nanoholes in a metal screen can perform the function of a lens: one-to-one imaging of a point source located a few tens of wavelengths away from the array to a point on the other side of the array. A displacement of the point source leads to a linear displacement of the image point. Complex structures composed of multiple point sources can be faithfully imaged with resolutions comparable to those of high numerical aperture lenes.
\end{abstract}


Nanohole arrays in metal screens have exhibited many interesting optical properties, most notably the extraordinary transmission of light through periodic [1] and quasi-periodic [2] nanoholes, polarisation conversion [3] and optical energy concentration $[4,5]$. Recently we have demonstrated that a quasi-periodic array of nanoholes in a metal screen can concentrate optical energy into hot spots and form subwavelength spots at distances a few tens of wavelengths away from the array [4, 5], when illuminated with plane waves (Fig.1(a)).

Here, we further demonstrate that such an array of quasi-crystal nanoholes in a metal screen can image a point source a few tens of wavelengths from the array into a spot on the other side of the array (Fig.1(b)). A displacement of the point source leads to a linear displacement of the image point, therefore the array can perform a one-to-one imaging function like a conventional lens. We show that complex structures composed of multiple point sources can be faithfully imaged with resolutions comparable to those of conventional lenes with high numerical apertures. Such a nanohole array lens may find important applications in circumstances where conventional optical lenes are not readily applied, like x-ray imaging, nano-optical circuits, etc.

(a)

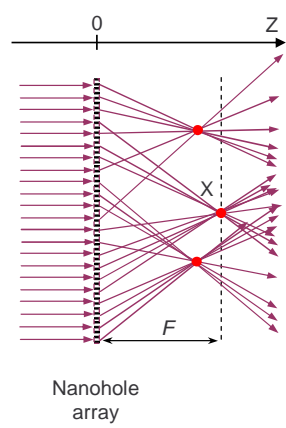

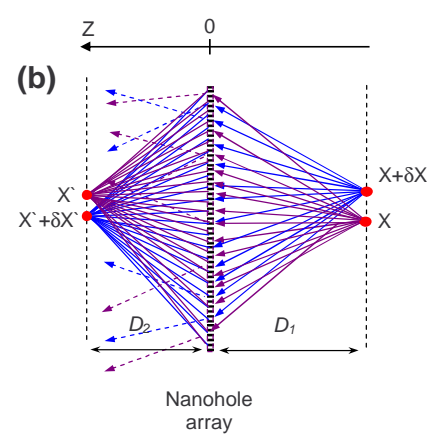

FIG. 1: Schematic diagrams illustrating a quasi-periodic array of nanoholes as a light concentrator converting a plane wave into hot-spot foci(a) and performing the function of a lens imaging two closely spaced optical point-sources $(x$ and $x+\delta x)$ to two spots $\left(x^{\prime}\right.$ and $\left.x^{\prime}+\delta x^{\prime}\right)$ on the other side of the array (b).

Although any array of small holes will create a diffraction pattern when illuminated by a point source, a careful choice of nanohole pattern is required for lensing applications. For instance, a regular $80 \times 80$ array of $200 \mathrm{~nm}$ holes (Fig.2(a)) will be self-imaged via the Talbot effect into a dense pattern of hot-spots and is not suitable as a lensing device (Fig.2(c)). However, a Penrose array of $200 \mathrm{~nm}$ holes (Fig.2(b))can at some distances create an isolated 
hot-spot of electromagnetic radiation as presented in Fig.2(d). The case of a Penrose array nano-hole as a lensing device will be investigated here in detail.
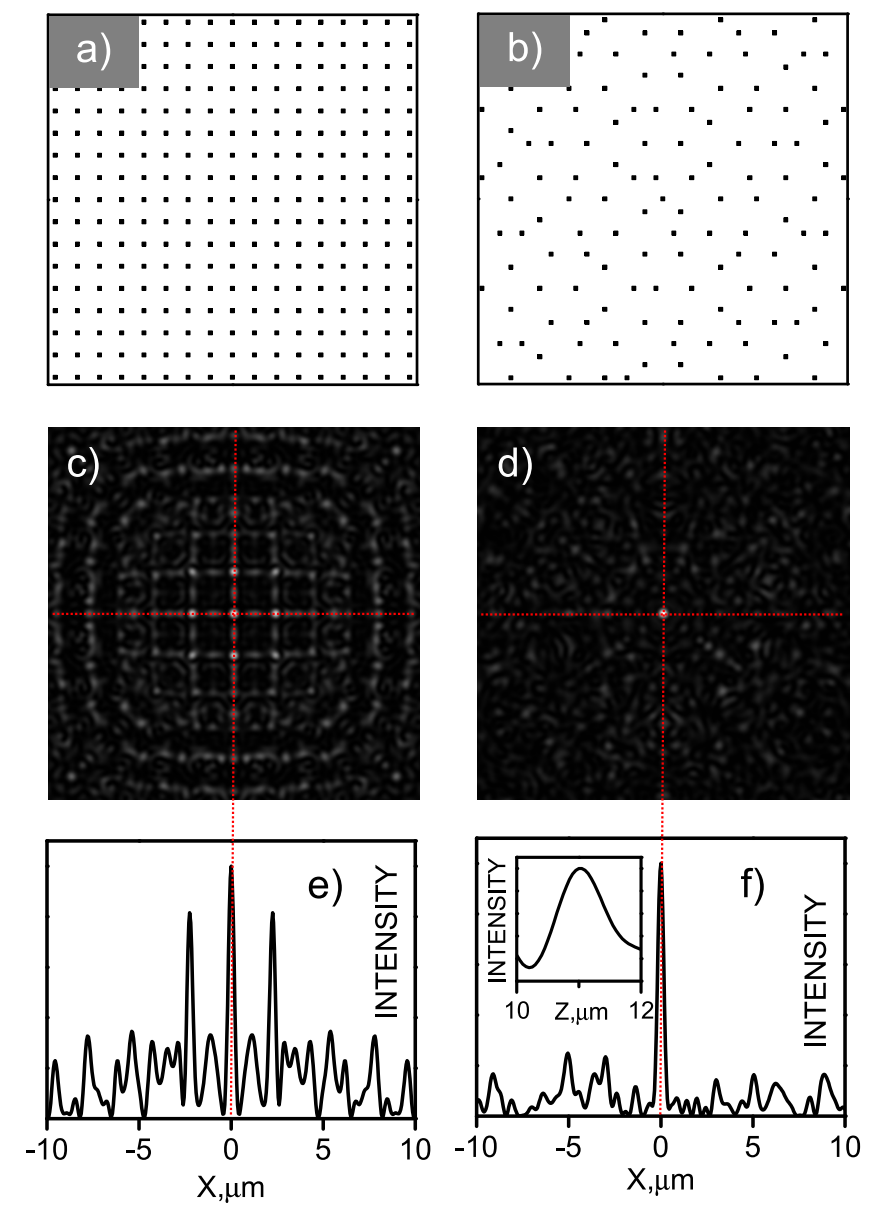

FIG. 2: Regular (a) and quasi-crystal (b) arrays of holes and $20 \times 20 \mu m^{2}$ fragments of their diffraction patterns, (c) and (d), respectively, at a distance $Z=11 \mu \mathrm{m}$ from the array. (e) and (f) show scans across the patterns' centers. In both cases a point light source with a wavelength $\lambda=660 \mathrm{~nm}$ is positioned at $Z=-11.4 \mu \mathrm{m}$ on the other side of the array. The inset to (f) shows the intensity of the hot-spot as a function of distance from the array

The nanohole array investigated had a Penrose-like quasi-periodic pattern of approximate 10-fold symmetry formed by $200 \mathrm{~nm}$ square holes, with the minimum separation between neighboring holes of $1.2 \mu \mathrm{m}$. The array contained about 14,000 holes and had an overall size of about $200 \times 200 \mu \mathrm{m}^{2}$. The hole pattern was designed according to algorithm described in Ref. [6].

The lensing properties of the quasi-crystal nanohole array were modelled using the 
Rayleigh-Sommerfeld scalar integration technique [7, 8]. This technique has proved to be consistent with boundary conditions when the observation points approach the diffraction aperture and is therefore suitable for our analysis. In all our calculations we assume that the object plane is located at a distance $z=-11.4 \mu \mathrm{m}$ from the plane of the array.

Fig. 3 shows characteristic different diffraction patterns created on one side of an array by a point source on the other side at different distances "Z" from the array. As the distance to the array varies, the diffraction pattern evolves dramatically, with a bright spot seen at the center of the image for some values of $\mathrm{Z}$. This bright spot can be seen as the focus of a lens and in this sense two parameters are important, the spot diameter at half height $d$ and the diameter $D$ of the "field of view" which we define as the dark area around the hot-spot where the intensity is less than $1 / e$ of the peak intensity at the "focus". For lensing applications one would like to minimize the focus $d$ and maximize the field of view $D$.

From Fig.3 one can see that the Penrose array can generate sub-wavelength hot-spots with various fields-of-view. For instance, with coherent illumination at a wavelength of $\lambda=660 \mathrm{~nm}$, at $Z=5 \mu \mathrm{m}$ and $Z=18 \mu \mathrm{m}$ foci with diameters of $360 \mathrm{~nm}$ are generated with fields of view of $2.8 \mu \mathrm{m}$ and $2.4 \mu \mathrm{m}$ respectively. A spot with a diameter as small as 200 $\mathrm{nm}$ can be generated within a smaller field of view (Fig.3, $Z=10.2 \mu \mathrm{m}$ ), confirming that subwavelength optical fields can be created in the far field without evanescent waves [4, 5]. The largest field of view, exceeding $D=20 \mu \mathrm{m}$ was found at $Z=11 \mu \mathrm{m}$ where the focus diameter was $380 \mathrm{~nm}$. The inset to Fig.2(f) shows the dependence of light intensity on the distance from the array of the $Z=11 \mu m$ focus. From here one can estimate tat the depth of focus (focal "waist") is $0.9 \mu \mathrm{m}$.

An essential property of a lens is the ability to image a point source and to distinguish between two neighboring points. This is illustrated in Fig.4. When the point source is moved within the object plane at $Z=-11.4 \mu \mathrm{m}$ from a starting position at $Y=0$ to $Y=400 \mathrm{~nm}$, the image spot moves linearly in the opposite direction in the image plane at $z=11 \mu \mathrm{m}$ from $Y^{\prime}=0$ to $Y^{\prime}=400 \mathrm{~nm}$, in a manner analogous to a conventional lens with approximately unitary magnification (see Fig.4, upper row ).

The imaging of two closely spaced point sources is illustrated for cases of both coherent and incoherent sources. Fig.4 (a-c) show images of coherent sources separated by 500, 600, and 700 nm respectively while Fig.4 (d-f) show images of incoherent sources separated by 400, 450, and 500 $\mathrm{nm}$. It appears that, as with a conventional lens, the resolving power 
of a nanohole array lens is better for incoherent illumination: according to the Rayleigh resolution criteria, coherent $660 \mathrm{~nm}$ point sources are resolved when spaced by $600 \mathrm{~nm}$, while incoherent point sources are resolved of a separation of $450 \mathrm{~nm}$. This may be compared with a conventional lens for which the coherent illumination resolution depends on numerical aperture $N A$ and is given by $0.77 \lambda / N A[9]$. Thus, the nanohole array shows an imaging ability analogous to a conventional lens with $N A=0.85$ for coherent illumination. Similarly, the resolution of a conventional lens for incoherent illumination is given by $0.61 \lambda / N A[9]$ and in this case the Penrose array has an effective numerical aperture of 0.89 .

The task of imaging a real object is illustrated in fig. 5 where we present images of multiple point sources of equal intensity of various configurations. The red dots indicate the expected positions of individual point sources images. The top row shows the case of coherent illumination while the bottom row shows that of incoherent illumination. The distances between points are $A B=600 \mathrm{~nm}, A C=700 \mathrm{~nm}, A D=1000 \mathrm{~nm}, A E=1140 \mathrm{~nm}, A F=$ $1000 \mathrm{~nm}, E F=420 \mathrm{~nm}$, the source wavelength $\lambda=660 \mathrm{~nm}$. One can see that under incoherent illumination, multiple point sources are faithfully imaged within a resolution of about $450 \mathrm{~nm}$. In the case of coherent illumination, as with a conventional lens, the resolution is degraded somewhat due to interference effects between different sources.

In summary, we have demonstrated that a quasi-periodic array of nanoholes can perform the function of a lens: i.e., the one-to-one imaging of a point source close to the array to an image on the other side of the array. Complex structures composed of multiple point sources can be faithfully imaged with resolutions comparable to those of high numerical aperture lenes. An interesting question emerges of whether lensing function ability with a resolution better than that of a conventional lens with a numerical aperture $N A=1$ could be achieved with a hole array. Although the Penrose hole array can creates subwavelength foci as small as 200nm in diameter (see Fig.3) the field of view is not sufficiently large for practical imaging applications. However, superoscillation theory specifies [10] that no principal limitation exists on the size of hot-spots or on the field of view. We therefore argue that our results provide an encouraging indication that more sophisticated nanohole patterns could generate small sub-wavelength hotshots with large fields of view, acting as true lenes. Here, the price to pay for high resolution in a large field of view will be a decrease in the power concentrated in the hot-spot relative to the power going into the unfocused side-bands located outside the field of view [11]. 


\section{Acknowledgments}

The authors would like to acknowledge the financial support of the EPSRC (UK), and fruitful discussions with E. Yablonovitch, N. Engheta, M. Denis and F. J. Garcia de Abajo. The authors thank Kevin MacDonald for help in preparing the manuscript.

[1] Ebbesen, T.W., Lezec, H.J., Ghaemi, H.F., Thio, T., Wolff, P.A., Nature 1998, 391, 667-669.

[2] Papasimakis, N., Fedotov, V. A., Schwanecke, A. S., Zheludev, N. I., and Garcia de Abajo, F. J., Appl.Phys.Lett.2007, 91, 081503.

[3] Krasavin, A.V., Schwanecke, A.S., Reichelt, M., Stroucken, T. Koch, S., Wright, E.M., Zheludev, N.I., Appl. Phys. Lett. 2004, 86, 201105.

[4] Huang, F. M., Chen, Y., Garcia de Abajo, F. J., and Zheludev, N. I., Appl. Phys. Lett. 2007, 90, 091119.

[5] Huang, F. M., Chen, Y., Garcia de Abajo, F. J., and Zheludev, N. I., J.Opt.A: Pure Appl. opt. 2007, 9, S285-S288.

[6] Lord, E. A., Ramakrishnan, K., and Ranganathan, S., Bull. Mater. Sci. 2000, 23, 119-123.

[7] Wolf, E. and Marchard, E.W., J. Opt. Soc. Am. 1964, 54, 587-594.

[8] Gillen, G. D. and Guha, S., Am. J. Phys. 2004, 72, 1195-1201.

[9] Born, M. and Wolf, E., Principles of Optics.

[10] Berry, M. and Popescu, S., J. Phys. A: Math. Gen. 2006, 39, 6965-6977.

[11] Frieden, B. R., Opt. Acta 1969, 16, 795-807. 


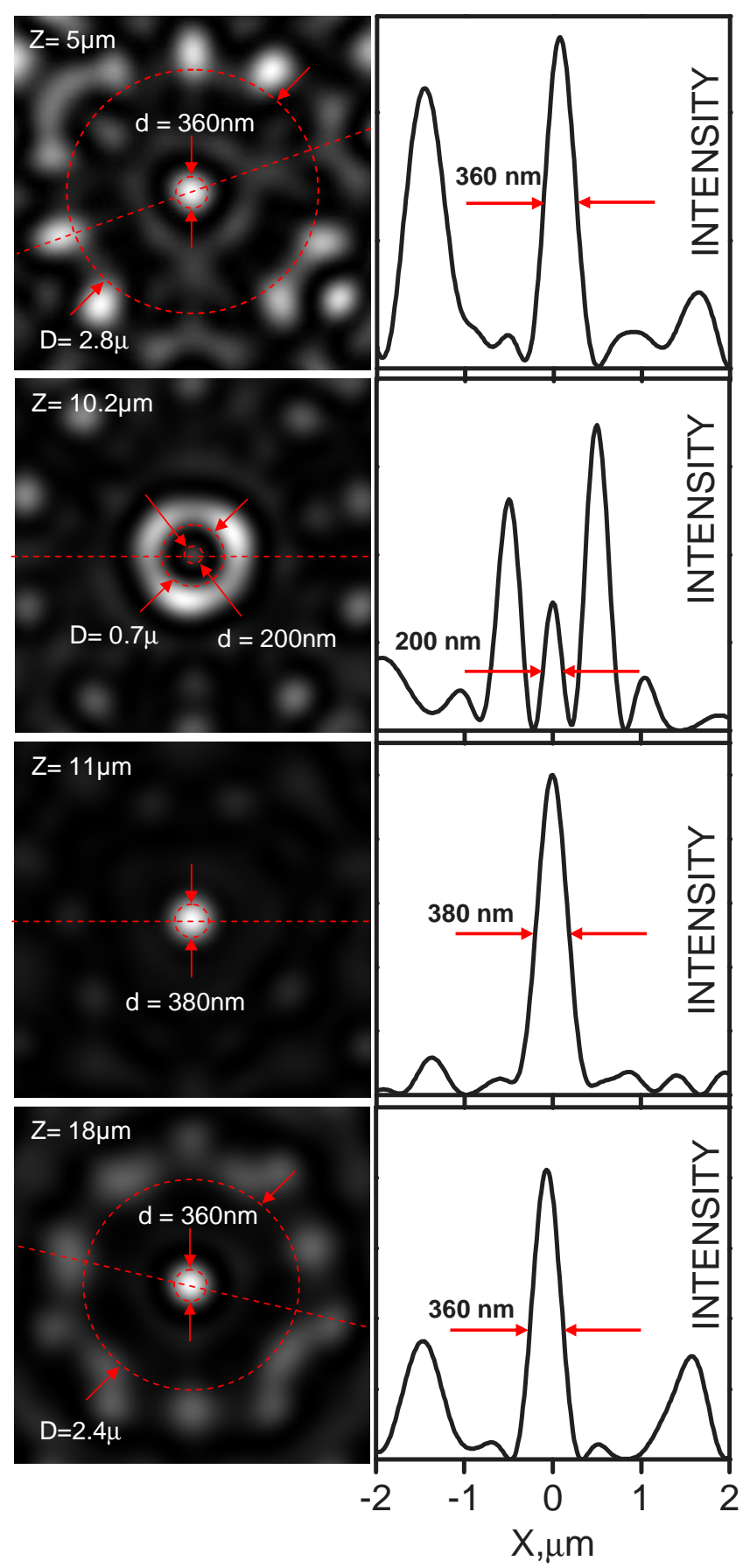

FIG. 3: Foci created at different distances $Z$ from a Penrose nano-hole array illuminated by a point source with $\lambda=660 \mathrm{~nm}$ positioned at $z=-11.4 \mu \mathrm{m}$. The dashed circles show "fields of view" and diameters of the foci 

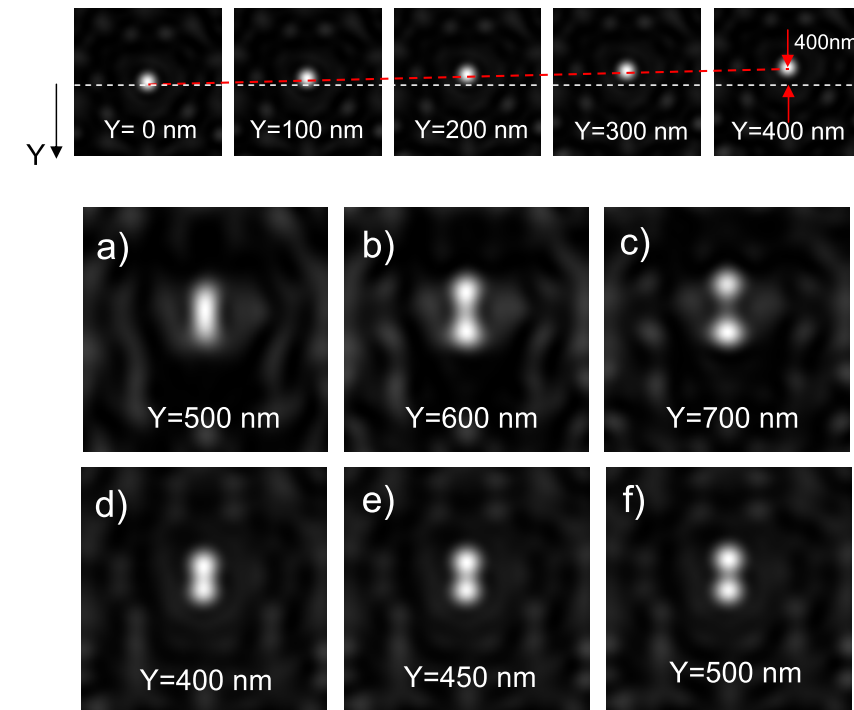

FIG. 4: Lensing properties of the Penrose nanohole array. The series of images in the upper row shows one-to-one imaging of a single point source as it is translated in the $Y$ direction in the object plane. Its image moves linearly in the image plane in the opposite direction. Figures (a-c) show images of two coherent $660 \mathrm{~nm}$ optical point sources separated by $Y=500,600$ and700 $\mathrm{nm}$ respectively. Figures (d-f) show images of two incoherent sources separated by $Y=400,450$ and500nm respectively. The optical point sources are positioned at $z=-11.4 \mu m$ and the image spots are in the image plane at $z=11 \mu \mathrm{m}$. In all cases the image size is $4 \times 4 \mu \mathrm{m}^{2}$
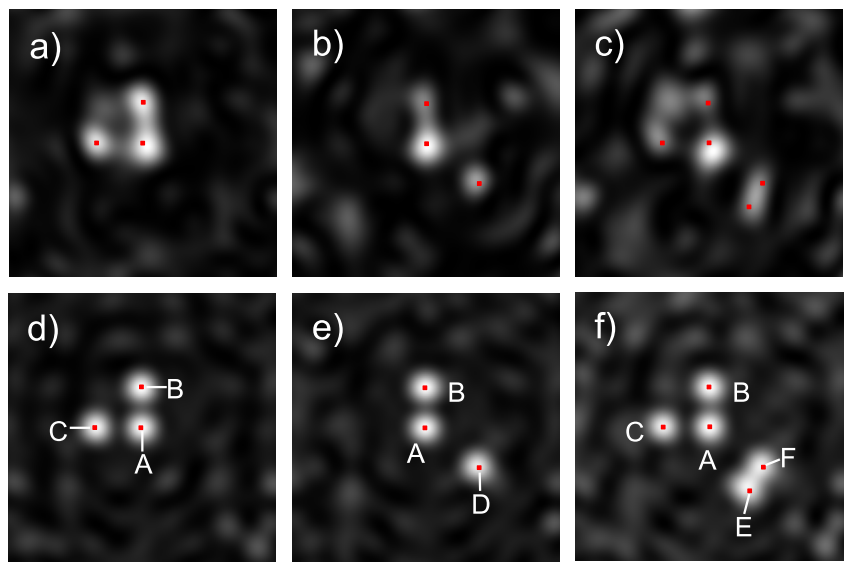

FIG. 5: Images of complex objects created by multiple point sources. The top row (a-c) shows images under coherent illumination while the bottom row (d-f) corresponds to incoherent source imaging. All images are $4 \times 4 \mu m^{2}$. 